\title{
Fundamental Use of Surgical Energy ${ }^{\mathrm{TM}}$ (FUSE): a curriculum on surgical energy-based devices
}

\author{
Amin Madani - Daniel B. Jones • Pascal Fuchshuber • \\ Thomas N. Robinson • Liane S. Feldman
}

Received: 25 April 2014/ Accepted: 26 April 2014/Published online: 18 June 2014

(C) Springer Science+Business Media New York 2014

The Fundamental Use of Surgical Energy (FUSE) is a new curriculum developed by the Society of American Gastrointestinal and Endoscopic Surgeons (SAGES). Surgeons and anesthesiologists can earn CME credits, nurses can earn CEUs, and those who master the curriculum content can obtain a certificate. But why is this needed?

Almost every surgical procedure, regardless of specialty or geographic location, involves the use of devices that apply energy to tissues. This practice has existed for thousands of years, whether it is cautery used by Egyptians in 3000 BCE to manage hemorrhagic shock [1] or Bovie's revolutionary electrosurgical unit introduced by Cushing into surgical practice in the early twentieth century [2]. Since their introduction, electrosurgical devices have become ubiquitous throughout operating theaters, endoscopy suites, and other procedural rooms. One of the most remarkable developments in surgery over the last two

For the SAGES FUSE Task Force: Sharon L. Bachman, L. Michael Brunt, Bipan Chand, Suvranu De, Warren S. Grundfest, Daniel M. Herron, Gretchen Purcell Jackson, Stephanie B Jones, Dean J. Mikami, Malcolm Munro, Chan W. Park, William S. Richardson.

A. Madani $(\varangle) \cdot$ L. S. Feldman

Department of Surgery, McGill University, 1650 Cedar Ave, D6-

257, Montreal, QC H3G 1A4, Canada

e-mail: amin.madani@mail.mcgill.ca

L. S. Feldman

e-mail: liane.feldman@mcgill.ca

\section{A. Madani - L. S. Feldman}

Steinberg-Bernstein Centre for Minimally Invasive Surgery,

McGill University Health Centre, Montreal, QC, Canada

D. B. Jones

Department of Surgery, Beth Israel Deaconess Medical Center,

Boston, MA, USA

e-mail: djones1@bidmc.harvard.edu decades has been the introduction of a large variety of complex and task-specific energy devices, incorporating novel technologies, newer designs, and advanced functions. Common examples include ultrasonic devices using mechanical energy, advanced bipolar electrosurgical devices with cutting blades and impedance measurement, and image-guided radiofrequency ablation instruments. Regardless of one's practice, the momentum in surgical energy innovation has transformed the operating room and endoscopy suite into a place with a wide array of technologies and tools.

The success of energy devices is largely due to their ease of use, diverse configurations and proven utility for hemostasis, tissue dissection, and ablation. However, this success poses safety challenges. Energy devices can lead to severe iatrogenic complications, including operating room fires, accidental tissue injury, and interference with other implantable medical devices (e.g., pacemakers, implantable cardiac defibrillators). One contributing factor is that the devices tend to be poorly understood by operators, regardless of their level of experience [3]. Many surgeons

P. Fuchshuber

The Permanente Medical Group, Kaiser Medical Center,

Walnut Creek, CA, USA

e-mail: pascal.fuchshuber@kp.org

T. N. Robinson

Department of Surgery, University of Colorado, Denver, CO,

USA

e-mail: thomas.robinson@ucdenver.edu 
do not consider themselves experts in the use of energy devices and have incomplete knowledge regarding their safe use. In order to use energy devices both effectively and safely, it is imperative to close this knowledge gap regarding device function, appropriate use, and safety. The medical literature provides many reports and anecdotes of iatrogenic injuries related to energy devices [4-14]. Approximately 600 operating room fires occur annually in the United States alone, a subset of which lead to disfiguring injuries and mortalities [4]. Between 1996 and 1998, 628 cases of burns due to electrosurgical devices were documented by the Food and Drug Administration [15]. This prompted the Emergency Care Research Institute (ECRI) to rank surgical burns and fires as a Top ten health technology hazard in 2012 [4]. The prevalence of bowel injuries due to electrosurgery during laparoscopic surgery is estimated at 1-2 per 1,000 patients [6], with high morbidity related to unrecognized injuries $[7,8,11,12]$. With over 2 million laparoscopic procedures done annually in the United States [16], this represents a significant safety issue impacting thousands of patients every year. Furthermore, the health-care costs are just as significant with hundreds of millions of dollars spent on medicallegal claims related to electrosurgical injuries each year $[17,18]$.

Despite using energy-based devices on a daily basis, surgeons and surgical trainees may not always be familiar with their basic principles and functions, which further exacerbate their potential danger. SAGES recently assessed the baseline knowledge of 48 of its leaders using an 11-item multiple-choice question examination, testing their knowledge of the principles and adverse events related to the use of energy devices [3]. The median number of correct answers was $59 \%$ (IQR 55-73\%). Thirty-one percent of SAGES leaders did not know how to correctly handle an operating room fire on the patient; $31 \%$ could not identify the device least likely to interfere with a pacemaker; $13 \%$ did not know that thermal injury can extend beyond the jaws of a bipolar instrument; and $10 \%$ thought that a dispersive pad should be cut to fit a child. What is even more impressive is that their baseline scores appear to be similar to that of junior surgical residents [19]. Yet, there exists no standard and validated training program to teach surgeons about energy devices. Most training, including that received during residency, is obtained from industry-sponsored education, mostly through sales representatives [20]. In fact, standard surgical textbooks and other educational resources in surgical education seldom contain information about energy devices. These findings reveal a profound unmet need in the surgical community: surgeons and surgical trainees lack sufficient understanding of the appropriate and safe use of energybased devices.
Table 1 Fundamentals of Surgical Energy ${ }^{\mathrm{TM}}$ (FUSE) curricular domains and objectives

\begin{tabular}{lll}
\hline & Curricular domains (10) & Objectives (63) \\
\hline 1 & Fundamentals of electrosurgery & 7 \\
2 & Mechanisms and prevention of adverse events & 20 \\
3 & Monopolar devices & 4 \\
4 & Bipolar devices & 5 \\
5 & Radiofrequency for soft tissue ablation & 4 \\
6 & Endoscopic devices & 4 \\
7 & Ultrasonic energy systems & 5 \\
8 & Microwave energy systems & 2 \\
9 & Energy devices in pediatric surgery & 3 \\
10 & Integration with other medical devices & 9 \\
\hline
\end{tabular}

In an effort to address this knowledge gap and safety issue, SAGES has developed the FUSE program. FUSE is the first comprehensive educational tool consisting of a curriculum in the basic science of surgical energy, developed by a multidisciplinary team of content experts (including surgeons, anaesthesiologists, nurses, and engineers) [3]. Similar to its "Fundamentals" counterparts [Fundamentals of Laparoscopic Surgery ${ }^{\mathrm{TM}}$ (FLS) and Fundamentals of Endoscopic Surgery ${ }^{\mathrm{TM}}$ (FES)], it consists of a web-based multimedia curriculum (also available in book format) and a validated certification examination. However, unlike FLS and FES, there is no hands-on training or assessment component. The curriculum covers ten domains and addresses 63 objectives (Table 1) [21]. This curriculum emphasizes safety for all members of the operating room, while keeping the content clinically relevant with practical information, regardless of subspecialty. The FUSE certification assessment was developed through a transparent process led by psychometrics experts to meet the requirements for a "high-stakes" examination to identify a candidate with the minimally acceptable competency [20].

Evidence accumulated to date suggests that FUSE has significant value for both surgeons and trainees. A didactic post-graduate course based on FUSE curriculum objectives was piloted at the 2011 SAGES meeting [3]. Twenty-seven participants took an 11-item pre-test multiple-choice examination of critical knowledge, followed by a 10 -item post-test assessment after the course that covered the same content. The median number of correct answers increased from $55 \%$ (IQR 46-82 \%) to $90 \%$ (IQR 70-90\%). The results of this study suggest that the FUSE curriculum improves short-term knowledge regarding function and safety of surgical energy devices.

Similarly, a 2-hour simulation-based electrosurgical course based on the FUSE curricular and assessment objectives was evaluated in junior surgical residents [19]. 


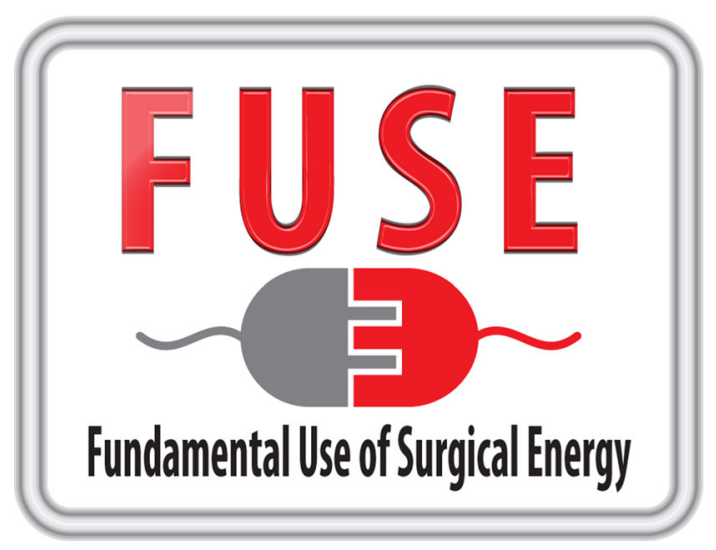

Fig. 1 The Fundamentals of Surgical Energy ${ }^{\mathrm{TM}}$ (FUSE) program

Fifty-nine first and second year trainees were assessed at baseline (pre-test), immediately post-curriculum and 3 months post-curriculum using separate 35 -item examinations. Median exam scores improved from $46 \%$ (IQR $40-54 \%$ ) to $84 \%$ (IQR 77-91\%) immediately after the curriculum. The results of this study reinforce the importance and utility of a formal curriculum on energy devices. Furthermore, the median 3-month follow-up examination score was $71 \%$ (IQR 60-86\%), demonstrating retention of knowledge, even months after the curriculum.

So why do we need FUSE? Perhaps a better way to phrase the question is: "Would you let yourself be operated on if you knew your surgeon did not receive formal training on the device used during your surgery?" SAGES' mission statement is to improve the quality of patient care through education, research, innovation, and leadership. The FUSE program embodies this objective by providing an opportunity for surgeons to acquire the expertise to use energy devices on patients both effectively and safely, and to deliver the best possible care. The evidence suggests that knowledge of energy devices can be taught at all levels, from residents early in their training to surgeons with decades of experience. The incorporation of FUSE into early surgical training will allow for trainees to learn and test on knowledge fundamental to the safe and optimal use of surgical energy devices. With the changing health-care environment and increasing demand for transparency and accountability, it is only a matter of time before credentialing bodies and administrators require certification of basic competency from surgeons and surgical trainees before using energy devices on patients for the first time. FUSE (Fig. 1) is a credentialing program developed by surgeons for surgeons and with the input of all team members in the operating room. It has shown its ability to close the gap in knowledge of function and safety of surgical energy devices as it pertains to surgeons and allied healthcare providers. The FUSE program is available to meet this need, and as of April 2014, 170 surgeons and allied health professionals achieved a passing score during beta testing of the exam. They are the first cohort of "FUSE certified" surgeons.

To learn more or sign up, visit http://www.fuseprogram. org.

Acknowledgments We acknowledge the SAGES FUSE task force for the development of the FUSE curriculum and certification examination.

Disclosures Drs Amin Madani, Daniel Jones, and Pascal Fuchshuber have no conflicts of interest or financial ties to disclose. Dr Thomas Robinson received research grants from Medtronics, Karl Storz Endoscopy and Covidien. Dr Liane Feldman is a consultant for Covidien and received an investigator-initiated research grant from Ethicon.

\section{References}

1. O'Connor JL, Bloom DA, William T (1996) Bovie and electrosurgery. Surgery 119:390-396

2. Schwaitzberg S (2012) Evolutions and revolutions in surgical energy. In: Feldman L, Fuchshuber P, Jones D (eds) The Fundamental Use Of Surgical Energy (FUSE) manual. Springer, New York

3. Feldman LS, Fuchshuber P, Jones DB, Mischna J, Schwaitzberg SD, Force FT (2012) Surgeons don't know what they don't know about the safe use of energy in surgery. Surg Endosc 26: 2735-2739

4. ECRI Institute (2010) Health devices: top 10 health technology hazards for 2011. ECRI Institute, Malaysia

5. Govekar HR, Robinson TN, Varosy PD, Girard G, Montero PN, Dunn CL, Jones EL, Stiegmann GV (2012) Effect of monopolar radiofrequency energy on pacemaker function. Surg Endosc 26:2784-2788

6. Nduka CC, Super PA, Monson JR, Darzi AW (1994) Cause and prevention of electrosurgical injuries in laparoscopy. J Am Coll Surg 179:161-170

7. Sankaranarayanan G, Resapu RR, Jones DB, Schwaitzberg S, De S (2013) Common uses and cited complications of energy in surgery. Surg Endosc 27:3056-3072

8. Agarwal BB, Gupta M, Agarwal S, Mahajan K (2007) Anatomical footprint for safe laparoscopic cholecystectomy without using any energy source: a modified technique. Surg Endosc 21:2154-2158

9. Gol M, Kizilyar A, Eminoglu M (2007) Laparoscopic hysterectomy with retroperitoneal uterine artery sealing using LigaSure: gazi hospital experience. Arch Gynecol Obstet 276:311-314

10. Kriplani A, Garg P, Sharma M, Lal S, Agarwal N (2008) A review of total laparoscopic hysterectomy using LigaSure uterine artery-sealing device: AIIMS experience. J Laparoendosc Adv Surg Tech 18:825-829

11. Polychronidis A, Tsaroucha AK, Karayiannakis AJ, Perente S, Efstathiou E, Simopoulos C (2005) Delayed perforation of the large bowel due to thermal injury during laparoscopic cholecystectomy. J Int Med Res 33:360-363

12. Darai E, Ackerman G, Bazot M, Rouzier R, Dubernard G (2007) Laparoscopic segmental colorectal resection for endometriosis: limits and complications. Surg Endosc 21:1572-1577

13. Peterson HB, Ory HW, Greenspan JR, Tyler CW Jr (1981) Deaths associated with laparoscopic sterilization by unipolar 
electrocoagulating devices, 1978 and 1979. Am J Obstet Gynecol 139:141-143

14. Willson PD, van der Walt JD, Moxon D, Rogers J (1997) Port site electrosurgical (diathermy) burns during surgical laparoscopy. Surg Endosc 11:653-654

15. Mann D (2000) Reducing the hazard of burns and bovie pads. Plast Reconstr Surg 106:947

16. Frost, Sullivan (1999) Market engineering research for the U. S. market for general surgery laparoscopy access and closure instruments. Medical and Healthcare Marketplace Guide. Frost and Sullivan, London, UK

17. Chandler JG, Voyles CR, Floore TL, Bartholomew LA (1997) Litigious consequences of open and laparoscopic biliary surgical mishaps. J Gastrointest Surg 1:138-145 discussion 145

18. Perantinides PG, Tsarouhas AP, Katzman VS (1998) The medicolegal risks of thermal injury during laparoscopic monopolar electrosurgery. J Healthc Risk Manag 18:47-55
19. Madani A, Watanabe Y, Vassiliou M, Fuchshuber P, Jones D, Schwaitzberg S, Fried G, Feldman L (2014) Does Simulation Improve Learning of the Fundamental Use of Surgical Energy ${ }^{\mathrm{TM}}$ (FUSE) Curriculum? A randomized-controlled trial in surgical trainees. Society of American Gastrointestinal and Endoscopic Surgeons, Salt Lake City

20. Feldman LS, Brunt LM, Fuchshuber P, Jones DB, Jones SB, Mischna J, Munro MG, Rozner MA, Schwaitzberg SD, Committee SF (2013) Rationale for the fundamental use of surgical Energy (FUSE) curriculum assessment: focus on safety. Surg Endosc 27:4054-4059

21. The SAGES Manual on the Fundamental Use of Surgical Energy (FUSE) (2012) Springer, New York 